\title{
Tableaux croisés chez Mercier et Rutlidge : le peuple de Paris et le plébéien anglais
}

\section{Raymonde Monnier}

\section{(2) OpenEdition \\ 1 Journals}

Édition électronique

URL : https://journals.openedition.org/ahrf/2234

DOI : 10.4000/ahrf.2234

ISSN : 1952-403X

\section{Éditeur :}

Armand Colin, Société des études robespierristes

\section{Édition imprimée}

Date de publication : 1 mars 2005

Pagination : 1-16

ISSN : 0003-4436

\section{Référence électronique}

Raymonde Monnier, «Tableaux croisés chez Mercier et Rutlidge : le peuple de Paris et le plébéien anglais », Annales historiques de la Révolution française [En ligne], 339 | janvier-mars 2005, mis en ligne le 27 avril 2006, consulté le 24 avril 2022. URL : http://journals.openedition.org/ahrf/2234 ; DOI : https://doi.org/10.4000/ahrf.2234

Ce document a été généré automatiquement le 24 avril 2022.

Tous droits réservés 


\title{
Tableaux croisés chez Mercier et Rutlidge : le peuple de Paris et le plébéien anglais
}

\author{
Raymonde Monnier
}

1 Les images du peuple dans la deuxième moitié du xvIII ${ }^{\mathrm{e}}$ siècle renvoient à des constructions sociales et identitaires complexes, qui conjuguent appartenance sociale et dimension nationale, où les représentations des élites et des moralistes croisent les constructions littéraires et administratives. Elles témoignent à la fois du poids de la mémoire réelle ou imaginaire et de la présence vivante d'un sujet turbulent qui s'impose dans la grande ville, dans la ville capitale, comme partie prenante de

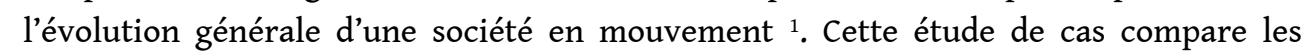
représentations de deux écrivains des Lumières inégalement connus et étudiés, Louis Sébastien Mercier et Jean-Jacques Rutlidge, d'un point de vue doublement comparatif, puisqu'il s'agit de tableaux parallèles sur le peuple de Paris et celui Londres, dans Le Babillard de Rutlidge (1778-1779) et le Tableau de Paris de Mercier (1781). C'est alors qu'on peut saisir, dans l'accélération des tensions, le regard et la parole moraliste dans leur crépuscule, pour reprendre les termes de Cyril le Meur dans une étude récente consacrée à quelques moralistes français de la fin du XVIII ${ }^{e}$ siècle $^{2}$.

2 L'analyse sémantique sera menée ici "au ras des mots ", à ce qu'ils signifient, aux images et aux préjugés qu'ils véhiculent chez deux auteurs des secondes Lumières, qui connaissent bien la civilisation anglaise, et ont pris part, chacun à sa façon, aux débats et aux combats de la Révolution française. L'étude comparative du vocabulaire des deux écrivains se justifie d'autant mieux qu'on sait que le sens politique du mot "peuple » émerge à l'Assemblée nationale avec l'expression représentants du peuple, par l'effet du colinguisme latino-anglo-français : par la «reprise à la Révolution du sens de poblo, forme initiale du français peuple dans les Serments de Strasbourg (842), sous l'influence de l'anglais people (13e siècle) lui-même emprunté à l'ancien français pople (du latin populus) par l'intermédiaire de l'anglo-normand poeple, people " ${ }^{3}$. En même temps qu'elle accompagne la promotion du mot dans son sens politique, l'expression proposée 
par Mirabeau, qui deviendra une des vedettes de la période révolutionnaire, marque une étape décisive dans l'évolution des institutions et des représentations politiques en France.

3 Le mot plébéien(s), que Mercier prend pour titre du chapitre 232 du Tableau de Paris pour comparer le peuple de Paris à celui de Londres, est dans le vocabulaire républicain une notion socio-politique située dans le même champ sémantique que peuple et citoyen : on est, à la veille de la Révolution, dans l'usage conceptuel de la liberté en marche. Mercier l'emploie à propos de la liberté politique, dans un chapitre qui suit celui sur la Liberté religieuse, où il célèbre la tolérance due aux Lumières : « La liberté religieuse est au plus haut degré possible à Paris ». Ce qui ménage un habile contraste avec le début du chapitre suivant: "Mais aussi la liberté politique, qui serait encore plus précieuse, à Paris, est nulle. Je suppose que l'on veuille ressusciter parmi nous le nom de plébéiens : eh bien! cela serait impossible, parce qu'il n'y aurait aucun sens attaché à ce mot. On ne pourrait pas dire le plébéien français, ainsi qu'on dit le plébéien anglais ».

4 Le terme plébéien n'appartient pas au lexique habituel de Mercier; il ne l'emploie que dans ce chapitre du Tableau de Paris, un chapitre publié dans la première édition, celle de 1781, et où il s'inspire de toute évidence du journal de son ami Rutlidge, Le Babillard. Ces emprunts faits par Mercier, que Rutlidge dénonce un peu plus tard, sont d'ailleurs à l'origine de leur brouille ${ }^{4}$. Il n'y a pas besoin de présenter Mercier et son Tableau de Paris. En revanche, Jean-Jacques Rutlidge est beaucoup moins connu des historiens, et son périodique Le Babillard n'a pas beaucoup intéressé les chercheurs ${ }^{5}$. C'est un journal inspiré du Tatler et du Spectator d'Addison et Steele que Rutlige prend pour emblèmes, et qui est assez fidèle à l'objet annoncé "Ouvrage littéraire, politique et moral ». Son auteur est un homme de lettres proche de Mercier par ses intérêts et ses combats littéraires ; il a déjà publié des comédies satiriques, des essais et des romans de mœurs ${ }^{6}$.

5 Pour situer rapidement le personnage, bien qu'il se fasse appeler James Rutledge et s'intitule lui-même "gentilhomme anglais », le Chevalier de Rutlidge est français et né en France de parents français. Mais son père, qui était armateur à Dunkerque, descendait d'une famille catholique irlandaise et avait soutenu l'expédition du Prétendant Charles Édouard Stuart en 1745, ce qui lui valut d'être anobli. Bien qu'il soit fils d'un jacobite et qu'il ait servi un moment dans un régiment irlandais, Jean-Jacques Rutlidge ne partage pas les sentiments de cette communauté d'émigrés; tolérant en matière religieuse, il est pour l'amitié franco-anglaise. C'est aussi un écrivain engagé dans un combat militant pour faire connaître la civilisation et la littérature anglaise ; il cherche à rapprocher les deux nations en traitant avec humour de leurs préjugés respectifs.

Dans le Paris des Lumières, Rutlidge représente le versant militant pour l'indépendance des auteurs et l'originalité des œuvres littéraires. Il ferraille avec humour contre tout ce qui brime ou bride l'expression des artistes et des poètes, défend Shakespeare contre Voltaire et soutient Mercier dans sa querelle avec les Comédiens français. Comme Rousseau, il le fait de manière paradoxale pour un auteur désireux de faire carrière dans les Lettres, en rompant les codes convenus du monde littéraire parisien. C'est à la notoriété de scandale d'une de ses pièces, Le Bureau d'esprit, que fait allusion Mercier dans le Tableau de Paris: "J'ai vu les Encyclopédistes n'accorder du mérite, des talents et même de l'esprit, qu'aux gens de leur parti, et vouloir bientôt juger tous les arts, même les plus éloignés de leurs connaissances. Ils ont donné prise sur eux par ce 
ridicule outré : ils ont été ridiculisés à leur tour, pour avoir manqué d'esprit, en voulant dominer tous les esprits. On a ri à leurs dépens, et l'on a très bien fait " ${ }^{7}$. Cette comédie à clefs publiée à Liège en 1776, éclaire de l'intérieur pour s'en moquer le salon mythique de Mme Geoffrin. La mort peu après de l'héroïne principale fit juger l'ironie de fort mauvais goût par les philosophes; en butte à la fureur de d'Alembert, Rutlidge fut détenu un temps au secret, ce qui dut l'inciter à plus de modération. Reste que le Chevalier de Rutlidge campe dans le Paris de la fin de l'Ancien Régime la figure de l'homme de Lettres républicain, adversaire déterminé du monopole de l'esprit, et soucieux de la liberté de ses choix dans une société qu'il voudrait plus ouverte aux idées et à la culture anglaise.

7 Parfaitement bilingue Rutlidge est, par ses œuvres littéraires et ses théories sociales, un médiateur engagé entre le milieu culturel parisien et le monde anglophone. Les transferts dans l'espace franco-allemand ont été bien documentés grâce aux enquêtes collectives dirigées par Michel Espagne et Michael Werner et, pour la période 1770-1815, par Hans-Jürgen Lüsebrink et Rolf Reichardt ${ }^{8}$. Il n'en est pas de même des échanges culturels franco-britanniques, en dehors des travaux d'olivier Lutaud sur l'édition de textes canoniques de la première révolution d'Angleterre à la Révolution française 9. Rutlidge, dont les œuvres sont moins connues, développe sous la Constituante une conception originale du gouvernement civil, en appui sur la théorie des États libres, et devient une personnalité en vue du Club des Cordeliers et du mouvement républicain de $1791{ }^{10}$. C'est un moraliste républicain, grand admirateur d'Harrington et de l'œuvre politique de Rousseau et de Montesquieu. Il publie en 1786 un éloge de ce dernier en soulignant sa filiation avec les penseurs de l'humanisme civique, de Machiavel à Harrington, et développe par la suite ses idées politiques dans ses journaux ${ }^{11}$. S'il fait un temps confiance à l'administration éclairée pour réformer la monarchie, il est cependant partisan de réformes plus radicales. Il compare le système politique, auquel on raccommode et refait sans cesse, mais qui n'offre qu'une masse antique imparfaitement réparée, au vaisseau de Thésée évoqué par Plutarque, objet des disputes des sophistes de la Grèce antique ${ }^{12}$. En républicain libéral et humaniste, Rutlidge n'est pas hostile au commerce ; il défend les valeurs de la sociabilité et de la communication dans un esprit ouvert et hospitalier qui imprègne ses essais périodiques.

8 Après le succès de sa Quinzaine anglaise (1776), qui peint les aventures d'un jeune Lord égaré dans les cercles de l'esprit et de la galanterie parisiens, il lance son Babillard «dans un moment où la politique occupe tous les esprits», en janvier 1778 quand la France s'engage aux côtés des États-Unis d'Amérique. L'auteur y met en scène divers correspondants fictifs qui discutent de questions d'économie politique, mais le journal traite aussi de critique théâtrale et littéraire et s'intéresse à des sujets d'actualité et aux mœurs. La forme adoptée, fidèle à l'esprit d'ouverture des Lumières, calque celle d'une pensée en mouvement où le dialogue est le mode privilégié d'expression dans la recherche de la vérité. Le Babillard qui a sans cesse de nouveaux objets de réflexion à offrir à ses lecteurs est ainsi placé au centre des controverses agitées dans le public. Le texte sur le caractère du Plébéien anglais, dont s'est inspiré Mercier, fait partie d'une série de Lettres de deux nouveaux correspondants, publiées de mai 1778 à février 1779, qui constitue un essai particulièrement réussi du journal de Rutlidge. Ses procédures énonciatives diffèrent sensiblement de celles de Mercier, dont le Tableau cherche à 
embrasser Paris tout entier, le désordre des chapitres et la multiplicité des thèmes étant sensés représenter l'incroyable diversité de la réalité parisienne ${ }^{13}$.

Dans Le Babillard l'échange épistolaire de deux Voyageurs, un Français en visite à Londres et un Anglais de séjour à Paris, qui font part de leurs impressions, permet au journaliste de composer un tableau vivant et contrasté des mœurs des deux capitales ${ }^{14}$. Il donne chemin faisant une esquisse du génie de la langue anglaise pour exalter ses poètes et compare quelques auteurs célèbres des deux pays; un examen tout en nuances qui entraîne le lecteur de l'étude de quelques vers du Paradis Perdu de Milton aux «beautés intraduisibles » de La Fontaine. L'éditeur commente la peinture que se renvoient les deux Voyageurs en conservant une distance critique; son but est de détruire les préjugés entretenus de part et d'autre sur les mœurs des deux peuples et des deux villes, pour instaurer un échange plus authentique : «je veux plutôt arrêter l'œil de la raison, sur les motifs \& la convenance d'une foule d'usages disparates, que nous copions les uns des autres avec fureur, \& que d'autres fois nous sifflons sans examen ${ }^{15}$.

10 Le rejet de l'artifice et le jeu des apparences qui ruinent tout espoir de restauration des valeurs républicaines est traité par l'ironie, qu'il soit question de la monotonie qu'engendre à Paris l'empire de la mode, de la vanité des titres de noblesse ou des travers du peuple caméléon des courtisans. Le dialogue du Voyageur anglais avec un Marquis qui illustre l'esprit français, permet de forcer le trait contre ceux qui « tantôt rampent comme des esclaves, \& tantôt s'enflent d'une manière pitoyable, comme des parvenus », et de conclure en moraliste : «Comme en fait de mœurs, l'imitation prend toujours sa source d'en haut, je présume que je trouverai en descendant, beaucoup de copies des objets qu'il m'a fait connaître ${ }^{16}$. La corruption progressive due à l'art des perruques rapportée au dénombrement des Friseurs de la capitale éclaire par contraste les mœurs simples des Peuples nouveaux qui font honneur au ministre qui en est à Paris l'incarnation, à ce Philosophe justement respecté, \& à ce Politique révéré, que nous possédons parmi nous ${ }^{17}$.

11 L'introduction de dialogues fictifs avec des Parisiens anonymes permet au Babillard d'avancer des arguments en conservant une certaine neutralité, tandis que ses correspondants poussent la critique sur les sujets de société, notamment en matière de politique et de religion. Le dialogue sur les mœurs joue sur les usages contrastés pour corriger ce qui est et suggérer ce qui devrait être: l'échange d'idées tend à perfectionner la société en aiguisant l'esprit critique dans la recherche du vrai. Le voyage est une expérience qui ouvre à la connaissance et à la conscience de soi et des autres ${ }^{18}$. Car nul n'est plus exclusif, remarque le Voyageur français, que celui qui n'a jamais bougé : «Je puis vous assurer, Monsieur, que vos Anglois ne sont guère moins ridicules, j'ai presque dit moins hommes que nous : rien ne ressemble autant, pour la gaucherie des opinions \& pour la crasse des préjugés, à un bon Parisien, que le Citoyen de Londres, qui y a passé toute sa vie. Les objets de leurs préoccupations diffèrent à la vérité ; mais ils sont encroutés l'un \& l'autre de la même nigauderie \& de la même obstination. Un Cockney prononce son sweet London! avec autant de dévotion \& d'enthousiasme qu'en met un gros Bonnet du Fauxbourg Saint-Honoré à s'écrier charmant Paris! Si ce dernier a l'impertinence de révoquer en doute qu'il puisse y avoir ailleurs du goût et de l'esprit, le premier met à peu près aussi stupidement en question, si l'on rencontre, hors de l'enceinte de sa Métropole, des plaisirs \& du sens commun; même affectation de part \& d'autre à louer les choses les plus communes \& à les ériger 
en merveilles " ${ }^{19}$. En observant les mœurs de la capitale britannique, le Parisien découvre sa propre culture et apprend à se connaître par comparaison en questionnant ses propres valeurs. Du sentiment patriotique des Français à l'amour-propre britannique, les pratiques sociales qui sont mises en scène reflètent une réalité qui donne matière à la réflexion morale et politique. La relation épistolaire des deux Voyageurs avec le Babillard est comme une métaphore de la vertu du commerce et de l'échange culturel, pour démentir les idées reçues.

C'est ce qu'entreprend Rutlidge, avec la Huitième Lettre du Voyageur Français à Londres, à propos du peuple londonien : «En général on est assez persuadé, \& surtout à Paris, que chez la Nation angloise le Peuple est plus Peuple qu'ailleurs : je veux dire qu'on l'y croit plus ignorant, plus emporté \& plus séditieux. Voilà un préjugé que j'oserai contredire » ${ }^{20}$. Dans les années 1770 les Français ont-ils toujours une si mauvaise opinion des Anglais? Pendant la guerre de Sept ans, une certaine littérature de propagande avait développé en France le thème de la barbarie des Anglais ${ }^{21}$. Mais l'opinion avait évolué depuis et un auteur comme Lefebvre de Beauvray, s'il était critique envers l'anglomanie de ses contemporains, adoptait en 1770 un discours patriotique mesuré quant aux mérites respectifs des deux nations, avouant même « que, s'il est en Europe un Peuple capable de disputer aux François la victoire en tous genres, c'est uniquement celui de la Grande-Bretagne " ${ }^{22}$. La controverse était plus politique que patriotique quand il en venait à l'histoire et à la constitution; pour un esprit conservateur partisan de la monarchie, la liberté ne peut s'acheter au prix de révolutions et de violences ${ }^{23}$.

Dans Le Babillard, Rutlidge raille volontiers l'anglomanie, mais s'en prend surtout, dans la lettre du Voyageur français, aux commentaires habituels et de parti pris sur la férocité de la nation anglaise. Mercier de son côté dénonce l'idée qui veut "que les Anglais mangent de la viande toute crue » ou que Londres soit une ville dangereuse. Le parallèle entre le peuple de Paris, habitué à céder à la « raison du fusil », et le peuple anglais, turbulent à Londres mais courageux au combat, traduit chez lui une préférence pour une politique mesurée capable de préserver le caractère national ${ }^{24}$. Dans une lettre précédente et sur un registre voisin, le correspondant de Rutlidge n'était pas insensible à la fierté des Anglais : « outre la grossièreté que le bas-Peuple a tout comme le nôtre, il montre une arrogance qui déplairoit peut-être moins à un Voyageur populaire \& philosophe, que l'allure rampante \& oblique qu'il a chez nous " ${ }^{25}$. Mercier déplore que le Parisien qui a «de l'esprit et des lumières" n'ait plus «ni force, ni caractère, ni volonté » et lui oppose « les vertus orgueilleuses » des États qui tiennent à une certaine rudesse. Il juge le bourgeois de Paris insouciant de ses intérêts politiques et « livré à une conduite mercantile ». L'ambiguïté du discours de Mercier dans le Tableau de Paris tient à un certain flou sémantique quant aux catégories qu'il met en scène - le peuple, le Parisien, le bourgeois - dans un discours où se mêlent les thèmes politiques et les topos du discours éclairé sur la ville. S'il se rassure en jugeant qu'une sédition est devenue pratiquement impossible à Paris, Mercier aimerait mieux voir le peuple, " comme à Londres, se battre à coups de poings, et s'enivrer à la taverne, que de le voir, comme à Paris, soucieux, inquiet, tremblant, ruiné, n'osant lever la tête, livré aux plus laides catins de l'univers, et incessamment prêt à faire banqueroute. Il est licencieux sans liberté... ${ }^{26}$.

Si on compare le texte de Rutlidge au chapitre Plébéiens de Mercier, plusieurs indices montrent que ce dernier s'est effectivement inspiré du Babillard ${ }^{27}$. Il suit les arguments quant aux causes empêchant ou favorisant au contraire la liberté politique, l'instruction 
et la capacité du peuple, à peu de chose près dans le même ordre et dans des termes identiques : la « liberté (indéfinie) de la presse ", «l'habitude et le pouvoir d'exprimer sans contrainte sa haine et son mécontentement"; [le peuple réuni/ le plébéien anglais] «juge, pour ainsi dire en corps, ses intérêts et ses guides : il a un caractère de raison et de rectitude"; une sûreté d'instinct/ un instinct sûr; notons encore la «bouche publique » d'où s'échappe « la vérité »; « la contradiction la plus légère ", « la moindre impatience » prise pour " attentat séditieux », « révolte illégitime ».

Mercier à l'évidence a suivi le texte de son ami à la lettre, est-ce à dire qu'il en a suivi l'esprit? Ces emprunts faits par Mercier ont le mérite de montrer ce qui les distingue. Arguments et tableaux croisés sur les avantages de la liberté politique, amènent les deux auteurs à conclure d'un côté à l'ignorance, au défaut de capacité et de patriotisme du peuple de Paris (Mercier), et de l'autre à la modération et au patriotisme éclairé de celui de Londres (Rutlidge). Est-ce anglomanie de la part de Mercier ? ou fierté du « vrai Parisien ${ }^{28}$ - ce que n'est pas Rutlidge. Si celui-ci tenait à dissiper les "préventions gallicanes ", Mercier, on le savait déjà, avait ses préjugés sur le peuple de la capitale et tient surtout à se distinguer des classes populaires. Son identité de vrai Parisien se construit par opposition à des catégories - le bourgeois, le peuple, la populace - qui se voient attribuer des nuances péjoratives «à usage unilatéral et en contradiction inégale ", selon un processus sémantique analysé par Reinhart Koselleck à propos de l'usage de certains couples d'antonymes ${ }^{29}$. Mercier fait tout au long du Tableau un grand usage du mot populace et écrit à propos du faubourg Marceau que "c'est un peuple qui n'a aucun rapport avec les Parisiens, habitants polis des bords de la Seine » 30 .

Le Tableau de Paris, donne du peuple une image pittoresque, toute en contraste ${ }^{31}$. C'est un peuple inculte, naïf et superstitieux, ivrogne et paillard, mais qui a une certaine délicatesse de goût, des dons et même parfois une sorte de génie créateur. Il est curieux de géographie et d'histoire et se passionne pour les premiers aéronautes. Mais il est avant tout badaud, il aime ce qui brille, va contempler les richesses des palais et des églises et s'anime au passage des processions et des défilés. D'où l'opinion communément reçue que le Peuple, en général, est plus éclairé à Londres qu'il ne l'est à Paris. Les travaux sur l'opinion et la culture populaire à la fin de l'Ancien Régime corrigent en partie le point de vue pittoresque et élitiste de Mercier. Analysant « l'irruption de la parole » dans la capitale, Arlette Farge a montré comment tous s'y autorisent à dire et à penser : « la curiosité publique n'est pas un trait de caractère, mais un acte qui fait entrer chacun en politique ». Dans sa diversité, la parole populaire témoigne de la capacité d'information et de communication du petit peuple, dont la curiosité cherche ailleurs que dans l'ostentation du cérémonial monarchique, la vérité d'un univers qu'il commence à s'approprier ${ }^{32}$.

Chez Mercier, le parallèle sémantiquement inégal entre les habitants de Londres et de Paris oppose le plébéien anglais, capable de former un corps éclairé et actif, au peuple de Paris, une multitude vouée à l'ignorance et à la passivité. À partir d'une remarque de vocabulaire sur le mot plébéien, le peuple de Paris est ainsi dévalué en bloc sur le plan politique : «Le plébéien n'existe pas à Paris : il est peuple, populace ou bourgeois! Il a des titres, des maisons, des privilèges ou des charges; mais il n'a point d'existence politique ». À Paris, le peuple manque d'énergie pour les choses publiques : «il se rend simple spectateur des opérations ministérielles. Il croit que le gouvernement est, comme le cours du soleil, physiquement déterminé par une nature invariable. Aussi la 
stupidité et l'ignorance politique sont le caractère de la multitude à Paris, plus que dans les autres pays de l'Europe ; et je n'en excepte aucun ». Le dernier paragraphe, ajouté dans l'édition de 1789 sur le bourgeois de Paris, montre que Mercier juge ses concitoyens du point de vue unilatéral et élitiste du républicain des lettres ${ }^{33}$. On serait tenté de lui répondre que c'est pourtant à Paris, en 1789, qu'est né le citoyen.

$\mathrm{Au} \mathrm{XVIII}^{\mathrm{e}}$ siècle, le mot plébéien avait une valeur positive dans le vocabulaire républicain classique par référence à la Rome antique, en relation usuelle avec patricien. Il était à mi chemin du registre technique pour désigner une catégorie socio-politique, et du registre affectif pour désigner l'appartenance. C'est Montesquieu qui a réintroduit l'usage du mot dans le lexique républicain pour désigner un corps politique organisé ${ }^{34}$. Dans l'Esprit des Lois, l'emploi du substantif est politique et soutient la démonstration de la distribution des trois pouvoirs dans la Rome antique, notamment dans les chapitres historiques du livre XI où on relève dix-neuf emplois (chap. 13 à 18), et où le mot est employé principalement avec des verbes d'action: les plébéiens voulurent (trois occurrences)/ pourraient/ augmentèrent/ demandèrent/ ôtèrent/ gagnèrent/ établirent/ prétendirent. Le terme sert à désigner une des différentes parties de la cité, et à distinguer ses compétences et son pouvoir, par exemple dans la distribution de la "puissance de juger »: "Les lois qu'on appela sacrées, donnèrent aux plébéiens des tribuns, qui formèrent un corps qui eut d'abord des prétentions immenses [...] La loi des Douze Tables modifia ceci [...] le corps des plébéiens, ou, ce qui est la même chose, les comices par tribus, ne jugèrent plus que les crimes dont la peine n'étoit qu'une amende pécuniaire. Il falloit une loi pour infliger une peine capitale [...] Cette disposition de la loi [...] forma une conciliation admirable entre le corps des plébéiens et le sénat » (XI, 18).

Chez Diderot, appliqué à l'Antiquité, le mot est synonyme de citoyen: "à Rome des esclaves, des affranchis, des citoyens ou plébéiens et des patriciens " ${ }^{35}$. Dans le vocabulaire des Lumières, la corrélation plébéiens/ patriciens est transposée sur le couple noble/ roturier. On trouve l'usage du couple nobles/ plébéiens dans Le Babillard, pour dénoncer la vanité attachée à Paris aux titres les plus fantaisistes : "Un homme n'est qu'un homme, sous quelque nom qu'il arrive au monde. Que l'on prenne cependant Nobles \& Plébéiens en masse, on verra que ce préjugé influe sur leur être ; on ne pourra s'empêcher de convenir que les premiers en reçoivent un sentiment d'élévation, \& que leurs ames ne s'imprègnent de ce vernis qu'on appelle honneur, \& qui remplace, à quelques égards, les principes \& la vertu [...] Le vrai Noble lui-même renoncera aux attributs qui servent à se distinguer, lorsqu'il verra qu'avec un peu d'effronterie on peut jouir \& s'environner de l'éclat extérieur qui est attaché aux titres ; ou plutôt cet éclat s'anéantira tout à fait " ${ }^{36}$. Chez le baron d'Holbach, dans La Morale universelle, le lexique de l'utilité joue sur les valeurs d'opposition pour gommer le préjugé social : «Il n'est pas douteux que le plébéien le plus obscur, dès qu'il est honnête et laborieux, ne soit un citoyen plus estimable que le noble inutile ou pervers, qui souvent se croit en droit de l'accabler de mépris : celui qui sert bien la patrie n'est jamais ignoble ou roturier $»^{37}$.

Le nom plébéien(s) reste d'un emploi relativement peu courant au XVIII ${ }^{\mathrm{e}}$ siècle, mais il est situé dans le champ sémantique de peuple et citoyen : dans le vocabulaire républicain, il dit l'appartenance sociale et la capacité civile au même titre que citoyen; il désigne un corps social et non une masse indifférenciée, et s'oppose à multitude. Si Montesquieu a remis le terme plébéiens à l'honneur, Rousseau a donné au terme citoyen une valeur 
politique et juridique marquée, en rapport avec la liberté politique et morale de l'individu. Dans la Lettre du Voyageur Français, Rutlidge reprend l'emploi républicain du mot plébéien à la manière de Montesquieu pour désigner un corps politique; son but n'est pas de marquer une quelconque supériorité de Londres ou de Paris, mais de faire réfléchir sur le rapport entre la constitution politique, la liberté et les mœurs. Il ne partage pas les préjugés de Mercier sur le peuple de Paris ou le caractère politique des vrais Parisiens ${ }^{38}$. Dans le Tableau de Paris, le plébéien anglais n'était évoqué que pour produire un effet de contraste, opposer deux peuples ou plutôt deux images du peuple. L'intention de Rutlidge était tout autre, puisqu'il visait à construire un consensus moral pour détruire les préjugés respectifs des Français et des Anglais. L'échange réciproque de points de vue sur les mœurs de Londres et de Paris leur apprendrait à se connaître et à réfléchir sur leur situation politique.

21 Que disait Rutlidge du plébéien anglais, sans faire d'ailleurs du terme une exclusivité anglaise? « en examinant avec attention, \& par-dessus toutes choses, en ne tirant des conséquences que des faits, je crois pouvoir avancer au contraire que la masse des Citoyens des classes inférieures, non seulement a ici moins de cette stupidité qui dans tous les autres Pays civilisés de l'Europe caractérise la multitude, mais encore moins de grossière barbarie \& de férocité aveugle ». Toute la Lettre du Voyageur Français visait à opposer les effets pervers du gouvernement de la monarchie absolue à ceux bénéfiques de la liberté civile et de la liberté d'opinion, en louant le caractère d'un peuple libre, injustement traité par les Français. On appréciera la conclusion modérée du républicain : «J'imagine, $\mathrm{M}$. le Babillard, que rien même n'est plus propre à éteindre l'esprit de faction, à adoucir un Peuple \& à le rendre équitable que la liberté civile. De l'aveu de tous ceux qui ont écrit sur ces profondes matières, on est bien plus à l'abri des commotions, quand on a atteint ce point difficile de la législation, que lorsque l'équilibre de l'autorité \& de l'obéissance se trouve détruit entre les Parties constituantes d'un État, par la coexistence d'un pouvoir abusif \& illimité \& d'une odieuse servitude ».

L'emprunt littéraire n'est sans doute pas une spécialité du XvIII siècle ; il est ici assez instructif quant aux théories morales et politiques de deux auteurs contemporains de la Révolution française. L'examen parallèle des textes de Mercier et Rutlidge, le traitement du terme plébéien chez les deux auteurs, montre comment sur un sujet aussi brûlant que celui de la liberté politique, les mêmes arguments sur ses bienfaits peuvent mener à propos de la même idée, de la même notion et de son champ sémantique, à des jugements et à des tableaux historiques contrastés. Tandis que s'annonce le grand mouvement d'émancipation de la Révolution, les deux écrivains cherchent chacun à sa manière à construire dans la société de leur temps un espace de liberté où construire la cité idéale, un point d'équilibre susceptible de projeter une image rassurante de la république. 


\section{ANNEXES}

Louis-Sébastien Mercier, Tableau de Paris, 1782 et [1789] : Plébéiens

«Mais aussi la liberté politique, qui serait encore plus précieuse, à Paris, est nulle. Je suppose que l'on veuille ressusciter parmi nous le nom de plébéiens : eh bien! cela serait impossible, parce qu'il n'y aurait aucun sens attaché à ce mot. On ne pourrait pas dire le plébéien français, ainsi qu'on dit le plébéien anglais. Le plébéien n'existe pas à Paris : il est peuple, populace ou bourgeois ! Il a des titres, des maisons, des privilèges ou des charges ; mais il n'a point d'existence politique : il n'a ni l'habitude ni le pouvoir d'exposer sans contrainte sa haine ou son mécontentement. Le plébéien anglais juge, pour ainsi dire en corps, ses intérêts et ses guides : il a un caractère de raison et de rectitude. Le peuple de Paris, pris en masse, n'a point cet instinct sûr qui démêle ce qui lui serait convenable, parce qu'il manque d'instruction, qu'il ne sait point lire, ainsi que le plébéien anglais.

Comme il ne jouit point de la liberté de la presse, il manquera longtemps de capacité ; il est voué à l'ignorance. Son patriotisme n'étant pas éclairé, il est nécessairement faible, on ne connaît que des saillies qui se refroidissent. Il n'a pas même la liberté de se livrer à ses affections : on redouterait peut-être ses applaudissements autant que ses murmures.

Paris enfin n'a point de bouche publique par où s'échappe le cri fort et direct de la vérité. Elle ne tonne jamais à l'oreille du souverain ; elle sort d'une manière timide et détournée du sein du petit nombre qui, supportant moins le fardeau des maux publics, voit avec plus d'indifférence les méprises du gouvernement.

Ainsi point d'activité, point d'énergie pour les choses publiques, parce que le peuple n'a ni le droit de parler, ni d'être écouté. Il sait très bien qu'on métamorphoserait en attentat séditieux, en révolte illégitime, la contradiction la plus légère, la moindre impatience ; et il se rend simple spectateur des opérations ministérielles. Il croit que le gouvernement est, comme le cours du soleil, physiquement déterminé par une nature invariable. Aussi la stupidité et l'ignorance politique sont le caractère de la multitude à Paris, plus que dans les autres pays de l'Europe ; et je n'en excepte aucun.

[On ne peut donc rien imaginer de plus sot que la manière dont un bourgeois parle des puissances voisines. Il arrange tout sur l'idée du syndic de sa communauté, et il prend la hiérarchie du commissaire, du lieutenant de police et du ministre, pour le modèle de tout gouvernement. Il ne conçoit pas pourquoi des républicains se mêlent si vivement de la chose publique ; il est disposé à les regarder comme des mutins, des séditieux, qu'un roi devrait morigéner, pour les rendre plus paisibles].»

Louis-Sébastien Mercier, Tableau de Paris, éd. Jean-Claude Bonnet, Paris, Mercure de France, 1994, t. I, chap. CCXXXII, pp. 582-584.

Jean-Jacques Rutlidge, Le Babillard, $n^{\circ} 81.20$ février $1779{ }^{39}$

Huitieme lettre du voyageur François à Londres

« Mon cher Babillard,

Notre correspondance n'a été aussi longtemps interrompue, que parce que j'ai cru à propos de la suspendre jusqu'à ce que la première croûte de mes préventions gallicanes 
fût brisée ; pardonnez-moi cette expression qui sent un peu le pays que j'habite. J'espère vous prouver que j'ai étudié le genre de Peuple au milieu duquel je vis, \& vous donner lieu d'augurer que dans les circonstances critiques où il se trouve, je suis pas à pas l'esprit politique, qui le soutient dans ses résolutions \& l'équité naturelle qui le distingue.

En général on est assez persuadé, \& surtout à Paris, que chez la Nation angloise le Peuple est plus Peuple qu'ailleurs : je veux dire qu'on l'y croit plus ignorant, plus emporté \& plus séditieux. Voilà un préjugé que j'oserai contredire : en examinant avec attention, \& par-dessus toutes choses, en ne tirant des conséquences que des faits, je crois pouvoir avancer au contraire que la masse des Citoyens des classes inférieures, non seulement a ici moins de cette stupidité qui dans tous les autres Pays civilisés de l'Europe caractérise la multitude, mais encore moins de grossière barbarie \& de férocité aveugle.

Quand j'avance ce paradoxe si propre à affliger notre vanité que tous les parallèles offensent, je ne prétends point dire que le Plébéien anglois isolé \& considéré dans son individu, ait plus de valeur que le Plébéien françois ; mais il est certain que l'influence de la liberté politique dont il jouit, que l'habitude \& le pouvoir d'exprimer sans contrainte sa haine \& son mécontentement, sa satisfaction \& son estime, que cette liberté indéfinie de la presse, qui provoque ici tout le monde à lire \& à disserter, lui donnent un air d'instruction \& de capacité qu'il n'a point chez nous. Je ne mettrai pourtant pas une trop grande valeur à cette écorce suspecte \& trompeuse. Ce ne sera point sur l'espèce d'habileté indigeste dont il lui arrive souvent de se revêtir, qu'il obtiendra une préférence dans mon opinion.

C'est lorsque ce Peuple est réuni ; quand il juge, pour ainsi dire, en corps ses intérêts et ses guides, qu'il a réellement un caractère de raison \& de rectitude, ou si l'on veut m'interdire à son égard des expressions que l'orgueil des classes supérieures croit devoir être réservées pour elles, une sureté d'instinct qui frappe \& qui étonne. Je vais surprendre bien davantage, en ajoutant qu'il y joint une modération dont la multitude n'est capable nulle part. En effet, mon cher Babillard, depuis l'expulsion des Stuart, il seroit difficile de citer une seule circonstance où la populace angloise ait commis des excès de faits, \& sur-tout où elle ait manqué à l'équité qui est dans ses principes. On pourroit en rapporter au contraire un grand nombre, où elle a fait justice \& manifesté un patriotisme éclairé, avec le fracas à la vérité \& le tumulte qui est son expression inévitable, mais sans danger \& sans désordre essentiel.

Les applaudissemens \& le chant même de victoire d'un Peuple, qui se livre à son contentement \& à ses affections, peuvent affliger les oreilles du despote orgueilleux. Concentré dans ses fastueux Palais, il veut être adoré en silence par des esclaves tremblants ; à plus forte raison la contradiction la plus légère \& la moindre impatience lui paroissent toujours l'attentat séditieux d'une révolte illégitime ; \& la vérité, lorsqu'elle est émanée de la bouche publique, offense sa fierté stupide. Est-ce un si grand malheur pour le Souverain qui la chérit \& qui cherche à la connoître, que de l'entendre parvenir jusqu'à lui, malgré la barrière que les lâches Courtisans veulent lui opposer, \& de l'apprendre avec sûreté par les mouvemens passagers \& sans périls de la masse libre \& indépendante de ceux qui lui obéissent ? N'est-ce point cette masse qui est la plus à portée de juger des maux publics \& la plus en droit de s'en plaindre ; puisque c'est elle qui en supporte le fardeau, \& qui placée loin du Trône, 
qu'environnent le mensonge, la flatterie, l'ambition \& la cupidité, voit les événemens \& sent le contre-coup des méprises d'un Conseil, où ces passions viennent s'asseoir.

J'imagine, M. le Babillard, que rien même n'est plus propre à éteindre l'esprit de faction, à adoucir un Peuple \& à le rendre équitable que la liberté civile. De l'aveu de tous ceux qui ont écrit sur ces profondes matières, on est bien plus à l'abri des commotions, quand on a atteint ce point difficile de la législation, que lorsque l'équilibre de l'autorité \& de l'obéissance se trouve détruit entre les Parties constituantes d'un Etat, par la coexistence d'un pouvoir abusif \& illimité \& à une odieuse servitude.

Plus les prérogatives d'un Peuple lui assurent une portion satisfaisante de cette même liberté, plus il est assuré que le cri de son mécontentement aura d'activité et d'énergie, \& moins il y mettra de passion \& de fureur. Plus il a droit de parler \& d'être écouté, \& moins il sera emporté \& séditieux. La conviction, qu'a le Peuple anglois qu'il est quelque chose, est le garant de sa modération; c'est la cause efficiente de cet amour de l'ordre, précieux à tous les Citoyens parce qu'il leur est favorable à tous; de cette espèce de police naturelle qu'ils y exercent entr'eux \& qui force à multiplier les Archers et les Janissaires dans ces contrées, où un Peuple qui n'a aucun droit ne peut point en connoître. C'est encore la force du principe d'équité soutenu, avec laquelle il a quelquefois exigé que les objets de la faveur fussent dévoués à sa vengeance, \& dédommagé les victimes de leurs brigues \& de leurs cabales, par les honneurs d'un triomphe d'autant plus flatteur qu'un cris impartial \& unanime le décernoit.

Les réflexions que j'ai faites à ce sujet ont été fortifiées par l'événement qui vient de vous occuper presqu'autant à Paris, qu'il a agité les esprits à Londres. Je connois assez bien la précipitation de nos pétulans Compatriotes, pour ne point douter que l'antipathie nationale ne leur ait fait hasarder les commentaires habituels sur la férocité \& la barbarie angloise, \& proférer d'odieux \& sinistres prognostics sur le sort de l'Amiral Keppel [...] ${ }^{40}$.

Je pense, M. le Babillard, avoir apperçu un des résultats les plus précieux de la constitution britannique. Il a deux faces : pour la partie exécutive, la sûreté d'être toujours bien obéi, \& pour les instrumens qu'elle emploie, la certitude de n'être jamais enveloppée dans le blâme encouru par l'Administration qui projette quelque fois mal, et dont l'ordre public les rend les Agens aveugles \& passifs. C'est incontestablement l'une des perfections les plus frappantes de la machine politique, lorsque le premier \& le plus essentiel des points de la Justice distributive est établi dans le service de l'Etat, par cette assurance où se trouve chaque individu dans sa position relative à son ensemble, de ne supporter jamais que le poids bien apprécié \& bien décidé de ses fautes réelles, \& d'obtenir infailliblement la récompense d'avoir rempli la tâche claire \& déterminée qu'on lui a imposée.

À Londres, ce premier Février.

Le voyageur françois » 


\section{NOTES}

1.Voir notamment Images du peuple au XVIII siècle, Paris, Armand Colin, 1973 ; Sociétés \& représentations, $\mathrm{n}^{\circ} 8,2000$, Le peuple en tous ses états, dir. par J.-L. ROBERT et D.

TARTAKOWSKY, $378 \mathrm{p}$.

2.Cyril Le MeUR, Les moralistes français et la politique à la fin du XVIII siècle, Paris, Honoré Champion, 2002, $617 \mathrm{p}$.

3.Josette ReY-DeBove, Gilberte GAGNON, Dictionnaire des anglicismes (Paris, Le Robert, 1981) ; Raymonde MONNIER, « Autour des usages d'un nom indistinct: "peuple" sous la Révolution française ", Dix-Huitième siècle, $\mathrm{n}^{\circ} 34,2002$, pp. 389-418.

4.Calypso ou les Babillards, t. II, p. 180, $n^{\circ} 26$ du 25 octobre 1784. Rutlidge accuse Mercier du plagiat de « lambeaux entiers » du Babillard, à propos de son allusion au vaisseau de Thésée dans Promenons-nous (chapitre où il fait aussi des emprunts à d'autres auteurs). Louis Sébastien Mercier, Tableau de Paris, éd. Jean-Claude Bonnet, Paris, Mercure de France, 1994, t. I, p. 425.

5.L'intérêt que Babeuf porte aux journaux et aux théories sociales de Rutlidge n'a pas échappé à Victor DALINE, qui a étudié leurs relations avant et pendant la Révolution (Gracchus Babeuf à la veille et pendant la Grande Révolution française (1785-1794), Moscou, 1976).

6.Le Babillard, 5 janvier 1778-30 avril 1779, 4 vol. Sur la vie et les œuvres de Rutlidge, voir Le Chevalier Rutlidge " gentilhomme anglais » 1742-1794, par Raymond LAS VERGNAS, Paris, Honoré Champion, 1932. Voir aussi l'introduction de Pierre PEYRONNET à l'édition critique de trois de ses comédies : Les Comédiens ou le Foyer, Paris, Champion, 1999. 7.Tableau de Paris, op. cit., t. I, pp. 467-468. Mercier reprend le titre Bureaux d'esprit dans un chapitre ironique sur les salonnières (ibid., chap. 532, t. I, p. 1471). La pièce de Rutlidge a été rééditée avec Les Comédiens ou le foyer, op. cit. Les habitués des salons bourgeois sont aussi tournés en ridicule dans le numéro 46 du Babillard (6e Lettre du Voyageur Anglais).

8.Transferts. Les relations interculturelles. XVIII ${ }^{\mathrm{e}}-\mathrm{XIX}{ }^{\mathrm{e}}$ siècles, Éditions Recherches sur les civilisations, 1988. Kulturtransfer im Epochenumbruch, Leipzig, Universitätsverlag, 1997.

9. Olivier LUTAUD, « Emprunts de la révolution française à la première révolution anglaise », Revue d'Histoire Moderne et Contemporaine, 37, 1990, pp. 589-607.

10.Sur la résurgence des thèmes républicains, voir Raymonde MONNIER, «Républicanisme et Révolution française », French Historical Studies, vol. 26/1, 2003, pp. 87-118.

11.James RUTLIDGE, Éloge de Montesquieu, Londres, 1786, 35 p. Reprint EDHIS, Montesquieu dans la Révolution française, Paris, Genève, 1990, vol. 1. Rutlidge fait en 1785, dans Calypso ou les Babillards, une analyse de la vie et des œuvres de Harrington, dont l'Oceana ( $\mathrm{n}^{\text {os }} 49$ et 50).

12.Le Babillard, $\mathrm{n}^{\circ}$ 87, du 20 mars 1779 (IV, p. 241). Voir Les Vies parallèles, Theseus, XXVII, Paris, Club français du livre, 1967, t. I, p. 19 (traduction d'Amyot).

13.Jean Marie Goulemot, « Le Paris des philosophes », Écrire Paris, Paris, Seesam, 1990, pp. 33-40.

14.Le Babillard, tomes II à IV. Rutlidge avait déjà publié à Londres en 1770 en anglais et en français en 1776 un Essai sur le caractère et les mœurs des françois comparées à celles des anglois.

15.Le Babillard, $\mathrm{n}^{\circ}$ 25, 5 mai 1778, t. II, p. 9. 
16.Ibid., $\mathrm{n}^{\circ}$ 40, 20 juillet 1778, t. II, p. 253.

17.Ibid., nº 43, 5 août 1778, t. II, p. 295. Allusion à Franklin qui est à Paris de 1776 à 1785. 18.Voir l'article « Voyage » de Daniel Roche, dans Le monde les Lumières, V. FERRONE, D. Roche (dir.), Paris, Fayard, 1999.

19.Le Babillard, $\mathrm{n}^{\circ} 43,5$ août 1778, t. II, p. 297.

20.Ibid., $\mathrm{n}^{\circ}$ 81, 20 février 1779, t. IV, p. 151-152.

21.David A. BELL, The Cult of the Nation in France. Inventing Nationalism. 1680-1820, Harvard U. P., 2001, chap. 3.

22.C.-R. LeFEBVRE de BeAUVRAy, Dictionnaire social et patriotique ou Précis raisonné de connaissances relatives à l'économie morale, civile et politique, Amsterdam, 1770, p. 26, article « Anglomanie ». Plusieurs entrées traitent des mérites respectifs des deux nations. L'auteur dit à l'article « Français » estimer sincèrement les Anglais, mais ne pouvoir « entendre de sang-froid tous ces éloges outrés, \& même exclusifs, qu'on donne tous les jours parmi nous au Peuple Britannique » (p. 172).

23.Ibid., p. 179, art. « Grande-Bretagne ». Il critique la constitution anglaise et le despotisme des monarques.

24.Tableau de Paris, op. cit., chap. 460, t. I, pp. 1273-76, Émeutes.

25.Le Babillard, $n^{\circ}$ 43, 5 août 1778, t. II, p. 298. Quatrième Lettre du Voyageur français.

26.Tableau de Paris, op. cit., chap. 20, t. I, pp. 61-65, Le bourgeois.

27.Ibid., chap. 232, t. I, pp. 582-584.

28.Pour Mercier « naître à Paris, c'est être deux fois Français ; car on y reçoit en naissant une fleur d'urbanité qui n'est point ailleurs » (ibid., chap. 718, t. II, p. 622). 29.Sur la dynamique de l'usage de ces figures linguistiques, qu'il qualifie de concepts antonymes asymétriques, voir Reinhart KOSELLECK, Le Futur passé. Contribution à la sémantique des temps historiques, traduction J. et M.-C. Hoock, Paris, Éd. E.H.E.S.S., 1990, pp. 191-232.

30.Tableau de Paris, op. cit., chap. 85, t. I, p. 217. La base Frantext fournit une fréquence élevée du mot populace dans l'ouvrage (47 occ.). Mercier vient en tête des auteurs pour la période 1778-1788, quant à la fréquence relative et absolue du mot. Sur le bourgeois, voir notamment le chap. XX (ibid., t. I, p. 61).

31.Jean-Louis VISSIERE, « La culture populaire à la veille de la Révolution d'après le Tableau de Paris de Mercier ", Images du peuple au XVIII ${ }^{e}$ siècle, op. cit. Voir aussi Daniel Roche, Le Peuple de Paris, Paris, Aubier Montaigne, 1981, 288 p.

32.Arlette FARGE, Dire et mal dire. L'opinion publique au 18e siècle, Paris, Seuil, 1992.

33.Pour Mercier, l'élite de la littérature est concentrée à Paris, qu'elle y soit née, ou que la capitale l'attire et la retienne " comme par enchantement ». Tableau de Paris, op. cit., chap. 301, t. I, pp. 782-788.

34.Dans la base Frantext, sur 29 emplois du mot chez Montesquieu (dont 24 dans l'Esprit des Lois), il s'agit toujours du substantif (le plus souvent au pluriel), mis à part deux emplois de l'adjectif dans les Considérations sur la grandeur des Romains : « des magistrats plébéiens », et « les principales familles patriciennes et plébéiennes ».

35. Réfutation d'Helvetius, 1774, p. 444.

36.Le Babillard, $\mathrm{n}^{\circ}$ 37, 5 juillet 1778, t. II, pp. 201-202. Seconde Lettre du Voyageur anglais. 37.La Morale universelle, 1776, p. 96.

38. "Paris a toujours été de la plus grande indifférence sur sa position politique. ", Tableau de Paris, op. cit., chap. 25, t. I, p. 75.

39.Le Babillard, t. IV, pp. 151-160. 
40.L'amiral Keppel, qui commandait la flotte anglaise au combat d'Ouessant en 1778, traduit en justice pour avoir manqué de résolution dans cette affaire, fut finalement renvoyé absous et comblé d'éloges par les Chambres. Rutlidge cite ce cas en exemple de la prudence du Gouvernement Britannique, qui s'assure de la fidélité des chefs des armées par l'examen de leur conduite et une justice impartiale.

\section{RÉSUMÉS}

Cette analyse sémantique des mots peuple et plébéien adopte un point de vue doublement comparatif: dans des tableaux parallèles du peuple de Paris et de Londres, deux écrivains inégalement connus et étudiés, Louis Sébastien Mercier et Jean-Jacques Rutlidge, donnent des représentations contrastées du peuple des deux capitales à la fin de l'ancien régime. Dans un chapitre du Tableau de Paris, le parallèle sémantiquement inégal entre les habitants de Londres et de Paris, oppose le plébéien anglais au peuple de Paris, une multitude vouée à l'ignorance et à la passivité. Rutlidge dans Le Babillard, croise le regard et la parole de deux Voyageurs en visite à Londres et à Paris, pour dissiper les préjugés sur les mœurs des deux capitales. Il s'agit dans la Lettre du Voyageur français, dont s'est inspiré Mercier, de combattre l'idée de la férocité des Anglais : en montrant les avantages de la liberté politique, il conclut à la modération et au patriotisme éclairé du peuple de Londres. Alors que s'annonce la révolution, les deux écrivains tentent de construire, chacun à sa manière en croisant les mots et les arguments, une image rassurante de la république.

Cross-Reading Mercier and Rutlidge : the Parisian "Peuple " and the English " Plebeian ". This semantic analysis of the words peuple and plebeian is a twofold comparative exercise : in parallel matrices of the people of Paris and London, Louis Sébastien Mercier and Jean-Jacques Rutlidge, two authors of differing stature and fame, provide contrasting representations of the people of the two capital cities at the close of the old regime. In a chapter of the Tableau de Paris, the semantically unequal parallel between the citizens of London and Paris opposes the English plebeian with the Parisian peuple, a multitude doomed to ignorance and passivity. Rutlidge in Le Babillard intercepts the glances and words exchanged by two Travellers visiting London and Paris in order to dispel prejudice in respect of the two cities. The Letter of the French Traveller, which Mercier took as his source, aims at undermining the idea of English ferocity: by highlighting the advantages of political freedom, he commends the moderation and enlightened patriotism of the people of London. As the age of revolution dawns, the two authors try to construct, each in his own way crossing words and arguments, a reassuring image of the republic.

\section{INDEX}

Mots-clés : peuple , plébéien, citoyen, liberté politique , Paris, Londres, Mercier , Rutlidge 
AUTEUR

\section{RAYMONDE MONNIER}

C.N.R.S.

49 Chemin de la Vallée aux Loups

92290 Chatenay-Malabry

ray.monnier@wanadoo.fr 